\title{
Un prix d'écriture pour les étudiants en médecine
}

\author{
Alexandre Wenger \\ PhD, chaire Médecine et société, Université de Fribourg
}

Dans nos universités, les étudiants en médecine sont essentiellement évalués sous forme de QCM. Dans ce système, les questions et les réponses sont pré-formatées, et les étudiants n'ont pas le choix des mots. Cocher une réponse par une croix ne requiert aucune compétence de formulation. Dès lors, est-il bien pertinent de faire écrire les étudiants en médecine?

Oui, trois fois oui! D'une part, parce que la pratique clinique est faite de relations singulières et différentes à chaque fois. Une relation thérapeutique efficace suppose une bonne compréhension mutuelle et la capacité du médecin d'adapter son discours à son patient. Or, écrire c'est choisir sa formulation, c'est prendre conscience de ses effets, c'est s'exercer au lien avec autrui.

\section{Les étudiants suivent un médecin généraliste pendant quatre jours.}

D'autre part, parce que l'écriture est une pratique réflexive, qui permet de mettre en perspective ses propres présupposés, ses motivations et ses difficultés. En somme, écrire exerce l'esprit critique sur soi et sur sa profession.

Enfin, parce que les médecins évoluent aujourd'hui dans un monde où la communication est omniprésente - dans les médias, sur les réseaux sociaux, entre collègues - et où la maîtrise de l'expression et le contrôle des messages que l'on veut transmettre sont devenus une nécessité.

Depuis 2012, dans le cadre du programme Médecine et société (Medical Humanities) de l'Université de Fribourg, les étudiants en médecine de $3^{\mathrm{e}}$ année effectuent un exercice obligatoire d'écriture. Cet exercice a été créé et pensé en complémentarité avec l'introduction dans le cursus d'un stage de médecine de premier recours. Les étudiants suivent un médecin généraliste pendant quatre jours. Ils rédigent ensuite le compte rendu circonstancié d'une rencontre avec un patient effectuée dans le cadre de ce stage. Leur texte doit répondre à un ensemble de directives formelles insistant sur la cohérence interne du récit, la construction d'ensemble, ou encore la pertinence des informations rete- nues. L'exercice amène les étudiants à réfléchir à leur propre rôle de (futur) praticien, ainsi qu'aux contextes sociaux, familiaux, économiques, etc. qui peuvent faciliter ou entraver la relation médecin-patient. Il s'agit donc d'un exercice intellectuel de mise en forme d'une observation, et non d'un épanchement émotionnel. Le texte est suivi par quelques lignes qui reprennent la situation décrite, mais sous la forme brève du dossier médical: le contraste entre les deux formes d'écriture doit apparaître clairement.

Certains étudiants ont d'abord été déconcertés par la liberté (apparente) laissée par cet exercice. Mais rapidement, ils nous ont aussi fait part de leur satisfaction de pouvoir écrire. Après réflexion, nous avons donc décidé d'introduire pour la première fois en 2016 un prix d'écriture récompensant le meilleur texte francophone et le meilleur texte germanophone.

Après une double lecture de tous les textes, le $\mathrm{Dr} \mathrm{PhD}$ Julien Knebusch et moi-même nous avons sélectionné les 3 meilleurs textes francophones et les avons envoyés, anonymisés, à un jury composé cette année du prof. Thierry Carrel (Directeur de la Clinique universitaire de chirurgie cardio-vasculaire, Inselspital, Berne), de Mme Claire Huguenin (chanteuse et musicienne, Fribourg) et du Dr Emmanuel Venet (médecin et écrivain, Lyon, France).

A l'issue de cette procédure, nous avons le plaisir de vous présenter ici le texte primé, à savoir «On a toujours fait avec» de M. Mickaël Lehmann. Les jurés ont notamment apprécié dans ce texte une écriture fine et sans pathos, un style fluide qui exprime la lourdeur, la colère sourde et la pudeur qui caractérisent les protagonistes, enfin une pensée clinique que l'on sent nourrie par un questionnement philosophique riche.

\section{Remarque}

La livraison germanophone du Bulletin des médecins suisses publie cette semaine le texte de Mme Catherina Holeczek, lauréate du meilleur texte en allemand. 


\section{«On a toujours fait avec»}

\section{Mickaël Lehmann}

Etudiant en médecine, $3^{e}$ année, Université de Fribourg

Un jeune homme avec un gros rhume. Une personne âgée qui souffre d'un mal de dos s'ajoutant à un genou déjà douloureux. Une autre femme qui tousse et dont le nez coule à cause de l'hiver. Les patients s'enchaînent et les pathologies semblent se répéter. Souvent, la vieillesse amène son lot de troubles et de douleurs, qui parfois ne veulent pas disparaître, et avec lesquels il faut apprendre à vivre.

Voilà le quotidien d'un cabinet à la campagne. On entend les voitures passer sous les fenêtres. Le vent souffle fort aujourd'hui. Les arbres se courbent sous les violentes rafales. La tempête approche. Le cabinet est ordinaire. Un bureau sur lequel s'empilent de nombreuses feuilles, et un ordinateur qui prend la moitié de l'espace, et dont les touches du clavier claquent tout au long de la journée. Une table d'examen se trouve à l'autre bout de la pièce, avec, à portée de main, les instruments nécessaires à la prise en charge variée des patients qui consultent un médecin généraliste. Je suis

\section{Il sait qu'un traitement contre cette tumeur lui demandera beaucoup d'énergie, une énergie qu'il n'a pas envie de gaspiller.}

assis à la gauche du Docteur, dans un coin de bureau sur lequel j'ai posé mon bloc-notes. Les patients arrivent, discutent quelque temps, se font examiner, puis repartent avec une ordonnance et un rendez-vous pour des contrôles quelques mois plus tard pour les plus âgés. Parfois, j'examine à mon tour des poumons ou un cœur, et interroge un patient pour m'exercer à l'anamnèse. Cependant, je reste généralement en retrait le long de l'entretien médical, passif, observant les patients et écoutant leurs soucis.

«Au revoir, passez une bonne journée!» dis-je en serrant la main de cette dame, dont les problèmes articulaires ne semblent pas vouloir se résoudre. Le médecin s'en va ensuite chercher le prochain patient dans la salle d'attente. J'en profite pour jeter un œil à son dossier médical sur l'ordinateur: Monsieur Bernard. Une longue liste d'antécédents cardiaques et une liste de médicaments tout aussi longue. Le motif de la consultation...

Il arrive le souffle un peu court, et boitant légèrement. Je me lève et lui serre la main. La septantaine, un pull jaune pâle qui contraste avec un pantalon noir monté jusqu'à la taille. Il porte des chaussures bien cirées. Il a certainement pris un soin particulier à s'habiller aujourd'hui. Il a les cheveux blancs et courts, un visage rond et orné d'une barbichette blanche taillé court elle aussi. De grandes lunettes fines laissent apercevoir des petits yeux bleu glacé, légèrement plissés, et marqués par les cernes qui lui creusent le regard. De petites dents dépassent d'une bouche fine et rose.

«Alors, comment allez-vous aujourd'hui?» lance le médecin. Cette question habituelle posée pour débuter l'entretien médical, et qui permet également de se faire une idée du problème primordial du patient, de ce qui le préoccupe principalement, de ce qui l'inquiète aussi. "Fatigué. Il faut que je me force pour aller boire mon café le matin. J'ai de la peine.» Il continue. «Je suis toujours allongé la journée. Et je lis.» Les journées n’ont pas l'air très passionnantes pour M. Bernard. Les jours se ressemblent, avec comme simple distraction une grande bibliothèque qui déborde de livres. Il a également de la peine à se mobiliser, car le souffle lui manque au moindre effort. Il a même besoin d'aide pour se lever du lit parfois. Il poursuit avec le motif de la visite. Une mammographie a été effectuée il y a 15 jours, et une biopsie la semaine passée. Les résultats sont connus depuis peu pour le Docteur: Carcinome du sein, avec un ganglion lymphatique axillaire atteint. Ce n'est qu'une confirmation des soupçons qui planaient jusqu'à aujourd'hui. $M$. Bernard accuse le coup, mais semblait déjà s'attendre à recevoir cette nouvelle. Ses paroles sont lentes, mais fortes. Elles s'ajoutent à une atmosphère qui semble s'alourdir, comme à l'approche d'un orage. Pour lui, pas question de faire quoi que ce soit avec cette nouvelle situation. Pas de traitement, pas d'autre examen.

Le Docteur reçoit un appel sur le téléphone à côté de l'ordinateur. Il décroche, s'excuse et sort de la pièce. M. Bernard m'explique alors le parcours de cette tumeur. Il est à Berne lorsqu'une infirmière remarque une grosseur sous son mamelon. Là, il fait une échographie. La suite des examens, c'est pour voir si ça s'agrandit, continue-il. Il l'avait déjà senti parfois sous la douche, mais n'y avait pas vraiment prêté attention. Il remarquait juste que le téton était un peu retroussé. Maintenant, il attend seulement de savoir comment la suite va se passer. J'écoute les paroles de cet homme, ses peurs, ses expériences de vie aussi, et j'éprouve un mélange de respect et de tristesse pour son histoire. «Je n'avais vraiment pas besoin de ça. C'est quand même 
pas de chance, vous savez.» La porte s'ouvre et le Docteur fait irruption dans le bureau.

Il continue son entretien après s'être à nouveau excusé. Je lève alors le regard et observe $M$. Bernard. Ses yeux sont légèrement humides et un peu rouges, comme irrités. Il renifle un peu. Le Docteur explique les différentes possibilités de prise en charge, qu'il faut que M. Bernard aille voir un oncologue pour discuter d'un éventuel traitement de cette tumeur, ou qu'il est également possible de faire une opération. Mais celui-ci ne veut rien entendre. La situation est plus complexe. Les traitements ne sont pas envisageables. «Si je reste sur le plateau, j'verrai rien du tout! J'ai quand même la poisse!» Il appréhende beaucoup la suite des événements, ce qui est tout à fait compréhensible. Son cœur est faible, et il aura sûrement de la peine à tolérer une anesthésie générale. Cela, $M$. Bernard s'en rend bien compte. Il sait qu'un traitement contre cette tumeur lui demandera beaucoup d'énergie, une énergie

\section{Il n'y a pas de bonne ou mauvaise façon}

de réagir, et personne ne peut se préparer à une telle nouvelle.

qu'il n'a pas envie de gaspiller. Il devient rouge. J'aperçois quelques larmes couler sur ses joues. «J'ai fait ce qu'il fallait pourtant!» dit-il d'une voix assurée. Il a dû changer son hygiène de vie, changer ses habitudes afin de ménager son cœur. Il est alors facile d'imaginer que ces efforts peuvent sembler vains maintenant, que cette tumeur pourra précipiter les choses. Mais il reste fier. Le menton levé, il affirme avec vigueur ses attentes auprès du médecin. «Ce qui est sûr, c'est que je n'veux pas de chimio!» Cela le médecin l'entend et le comprend. Après de nombreux séjours à l'hôpital, parfois de plusieurs mois, après les souffrances causées par ses problèmes de santé, $M$. Bernard ne veut pas encore passer les derniers moments de sa vie avec les traitements lourds de la chimiothérapie. Le diagnostic de carcinome invasif, avec de plus un ganglion touché, laisse peu de chance à un rétablissement sans une prise en charge complète. C'est un choix parfois difficile, mais pour M. Bernard, c'est déjà tout décidé. Pas question d'acharnement de la part des médecins. «Maintenant, il faut accuser l'coup.» Le Docteur demande alors si sa qualité de vie est encore bonne, si finalement, il lui reste les ressources suffisantes pour continuer à vivre. Ce dernier lui explique sa routine quotidienne. Il va boire un café le matin, et ensuite, quelques fois, sa sœur ou son ami vient lui rendre visite pour aller manger dehors. A l'exception de ces moments, c'est assez malheureux. Il ne lui reste que la lecture pour s'échapper quelques temps de la réalité. «Mais je n'ai plus envie de retourner à l'hôpital!» s'écrie-t-il. Puis il se mouche bruyamment dans son mouchoir en tissu à carreaux. Ses yeux sont rouges et humides. J'ai l'impression qu'il se retient de pleurer, qu'il essaie de rester digne. «Mais je ne veux pas de chimio! Merde!» Il craque pendant quelques secondes. Les larmes coulent sur son visage. Il n'y a pas de bonne ou mauvaise façon de réagir, et personne ne peut se préparer à une telle nouvelle. Quand le résultat tombe, c'est comme si le monde s'écroule autour de vous. Actuellement, toute sa vie gravite autour de la maladie. Il ne lui est plus possible de faire ce qu'il veut, d'aller où il veut, de vivre comme il le veut. Aux problèmes cardiaques s'ajoute le cancer. C'est trop à accepter. Le problème médical, bien que central, est pourtant relégué au second plan au profit de la souffrance émotionnelle qu'il engendre. L'essentiel du problème est là. C'est pour lui la peur d'une épreuve insurmontable à combattre, et la peur d'une mort lente et douloureuse.

Après avoir discuté de la suite de la prise en charge, et pris un rendez-vous chez un oncologue, le médecin accompagne M. Bernard à la porte. "Ça veut aller?» demande-t-il. «Il faut. J’arrive à me reprendre.» Il s'approche vers moi. Je lui souris et lui sers la main, en essayant de montrer toute la sympathie et la compassion qu'il m'est possible de transmettre. Il demande alors au Docteur de quelle façon il pourrait mourir qu'il a déjà entendu, par un autre médecin, que ce sera un sommeil plus long qu'un autre, qu'il n'y verrait rien. Il s'y prépare, il y pense souvent. "Ça va aller?» demande à nouveau le Docteur. «On fera avec. On a toujours fait avec.»

\section{Dossier médical}

Patient de 73 ans avec un carcinome canalaire invasif du sein droit avec adénopathie positive, accompagné d'anxiété et de signes de dépression. Le patient refuse tout traitement ou intervention. Antécédents de remplacement valvulaire aortique par une valve biologique, pontage de trois vaisseaux, pose d'un clip mitral sur insuffisance mitrale sévère. Patient également atteint d'une cardiopathie hypertensive dilatée et de fibrillation auriculaire chronique. Fraction d'éjection du ventricule gauche abaissée. Facteurs de risques cardiovasculaires: Hypertension artérielle, diabète de Type II, et ancienne obésité.

Médicaments: XARELTO $20 \mathrm{mg} 1 \times / \mathrm{j}$, ASPIRINE CARDIO $100 \mathrm{mg} 1 \times / \mathrm{j}$, METO ZEROK $50 \mathrm{mg} 2 \times / \mathrm{j}$, DIGOXINE 0,25 mg $1 \times /$ j, PERINDOPRIL $4 \mathrm{mg} 1 \times / \mathrm{j}$, TORASEMID 10 mg $2 \times / \mathrm{j}$, ALDACTONE $50 \mathrm{mg} 1 \times / \mathrm{j}$, METFIN $850 \mathrm{mg} 2 \times / \mathrm{j}$, SERTRALINE $50 \mathrm{mg} 1 \times / \mathrm{j}$, PANTOZOL $40 \mathrm{mg} 1 \times / \mathrm{j}$, ZOLPIDEM $10 \mathrm{mg} 1 \times / \mathrm{j}$, SERETIDE $2 \times / \mathrm{j}$. 